\title{
Gluon field digitization for quantum computers
}

\author{
Andrei Alexandru, ${ }^{1,2, *}$ Paulo F. Bedaque, ${ }^{2, \dagger}$ Siddhartha Harmalkar, ${ }^{2, \$}$ Henry Lamm $\odot,{ }^{2, \S}$ \\ Scott Lawrence, ${ }^{2, \|}$ and Neill C. Warrington, ${ }^{2, \pi}$ \\ (NuQS Collaboration) \\ ${ }^{1}$ Department of Physics, The George Washington University, Washington, D.C. 20052, USA \\ ${ }^{2}$ Department of Physics, University of Maryland, College Park, Maryland 20742, USA
}

(Received 16 July 2019; published 3 December 2019)

\begin{abstract}
Simulations of gauge theories on quantum computers require the digitization of continuous field variables. Digitization schemes that use the minimum amount of qubits are desirable. We present a practical scheme for digitizing $S U(3)$ gauge theories via its discrete subgroup $S(1080)$. The $S(1080)$ standard Wilson action cannot be used since a phase transition occurs as the coupling is decreased, well before the scaling regime. We propose a modified action that allows simulations in the scaling window and carry out classical Monte Carlo calculations down to lattice spacings of order $a \approx 0.08 \mathrm{fm}$. We compute a set of observables with subpercent precision at multiple lattice spacings and show that the continuum extrapolated value agrees with the full $S U(3)$ results. This suggests that this digitization scheme provides sufficient precision for noisy intermediate-scale quantum era QCD simulations.
\end{abstract}

DOI: 10.1103/PhysRevD.100.114501

\section{INTRODUCTION}

Quantum computers can attack problems in physics which appear intractable on classical computers [1]. Large-scale quantum computers would allow simulations of nonperturbative calculations of real time evolution and finite-density equations of state. For the foreseeable future, though, quantum computers will be limited to tens or hundreds of non-error-corrected qubits with circuit depths less than a thousand gates-the so-called noisy intermediate-scale quantum (NISQ) era. QCD simulations on quantum computers-especially in the NISQ eradepend upon formulating QCD in an efficient way.

Fermionic fields like quarks can be easily digitized as qubit registers by encoding their presence or absence in a given state [2-5]. This is evident from the few existing calculations performed on digital quantum computers [6-9]. The continuous nature of gauge fields precludes

\footnotetext{
*aalexan@gwu.edu

†bedaque@umd.edu

*sharmalk@umd.edu

\$hlamm@umd.edu

"srl@umd.edu

"ncwarrin@umd.edu
}

Published by the American Physical Society under the terms of the Creative Commons Attribution 4.0 International license. Further distribution of this work must maintain attribution to the author(s) and the published article's title, journal citation, and DOI. Funded by SCOAP ${ }^{3}$. such exact digitization without modification, although for analog quantum computers this is less of an issue [10,11]. Proposed solutions involve eliminating the bosonic fields using some model-dependent properties, truncating in occupation number, discrete spins, or extending to higher dimensions [12-28].

The situation is reminiscent of the pioneering days of lattice field theory when computer memory was limited and the cost of storing $S U(3)$ elements was prohibitive. Several attempts were made to replace the continuous gauge fields by a discrete set of values [27,29-36]. Quantum computation is presently in a similar situation where every qubit comes at a high cost. While more efficient parametrizations are possible they trade memory for computational difficulty; modern lattice QCD calculations represent each gauge link by nine complex numbers represented using a double-precision format that would require 1152 qubits. In contrast, the largest "crystal-like" discrete subgroup of $S U(3), S(1080)$ contains 1080 elements and would require only 11 qubits to store each link value.

Digitization typically reduces the symmetry of the model although schemes exist which preserve the exact gauge symmetry [21,25-28,37] although finite truncations of these schemes to low-lying energy eigenstates can sacrifice unitarity at high energies [37]. With this reduction, it is not a given that the original model is recovered in the continuum limit as the universality class of the lattice model may change [38-43]. For any discrete group, there is always a finite difference in the action, $\Delta S$, between 
the field configurations with the two smallest actions, as opposed to continuous groups where no such gap exists. This may lead to "freezing" at some critical $\beta_{f}$; that is, all field values except the identity (and gauge equivalents) are exponentially suppressed. For values of $\beta$ beyond $\beta_{f}$ the theory with the discrete group differs drastically from the continuous group and is no longer a reasonable approximation. This is a particular problem for asymptotically free theories like QCD, although there are some discrete system counterexamples [28]. The spacetime continuum limit where the lattice spacing $a$ approaches zero is obtained by making $\beta$ large but that is where the continuous and discrete group theories differ. This is not necessarily fatal: Realistic lattice calculations are performed on classical computers with a finite $a$ and extrapolated in a controlled manner to $a \rightarrow 0$. In these calculations, what is required is that $a$ is smaller than typical hadronic scales (e.g., the size of hadrons). Typically values used in state-of-the-art calculations are $\mathcal{O}(0.1 \mathrm{fm})$. This corresponds to $\beta \gtrsim 6$ when using the Wilson action in the so-called scaling region. While we do not anticipate our $S(1080)$ action to be equivalent to $S U(3)$ in the continuum limit, as we shall see, they are similar at finite lattice spacing. Therefore our goal will be to set up a framework where discrete groups can be used to reproduce $S U(3)$ results in the scaling region, such that practical quantum computations of $S U(3)$ with $a \approx 0.08 \mathrm{fm}$ could be performed with reduced resources. This is a first and necessary step toward a proposal for QCD on a quantum computer by showing that the simulations using $S(1080)$ have merit for rough $S U(3)$ calculations in the NISQ era. With this success, the next step in studying the feasibility of this procedure is to construct the quantum registers and primitive gates à la [44] where smaller discrete groups were investigated.

There were a number of early studies of the viability of crystal-like discrete subgroups of $U(1)[45,46]$ and $S U(N)$ $[29,31,33]$ gauge theories including with fermions $[47,48]$. While the discrete subgroups all have freezing transitions for the Wilson action, $\beta_{f}$ increases with the size of the subgroup. For $U(1)$, the $\mathbb{Z}_{N}$ theories have a $\beta_{f}$ in the scaling regime (in this case $\beta \gtrsim 1$ ) for $N>4$. $S U(2)$ has only three crystal-like subgroups: the binary tetrahedral, $\mathbb{B} \mathbb{T}$, the binary octahedral, $\mathbb{B O}$, and the binary icosahedral, $\mathbb{B I}$. Using the Metropolis algorithm with 100 measurements separated by 1000 updates, we refined the results of [29], finding that while $\mathbb{B} \mathbb{T}$ has $\beta_{f}=2.24(8), \mathbb{B O}$ and $\mathbb{B I}$ have $\beta_{f}=3.26(8)$ and $\beta_{f}=5.82(8)$ respectively, both deep in the scaling regime $\beta \gtrsim 2.2$. Hence, these two last groups can be used in lieu of $S U(2)$ for practical calculations.

The story changes for $S U(3)$. There are five crystal-like subgroups: $S(60), S(108), S(216), S(648)$, and $S(1080)$ designated by their number of elements. For all these, $\beta_{f}<6$, with the largest, $S(1080)$, being reported to have $\beta_{f}=$ 3.58 (2) obtained on a $2^{4}$ lattice [31]. Our own calculations on a $2^{4}$ volume with larger statistics for $S(1080)$ show a slightly larger value $\beta_{f}=3.935(5)$. In any case, it is evident that the $S(1080)$ theory with the Wilson action is inadequate to reach the scaling regime. Subsequent work [34] showed that extending the elements to include the midpoints between elements of $S(1080)$ was sufficient to push $\beta_{f} \approx 7$. However this requires more bits and sacrifices the group structure.

To overcome these limitations, attempts were made to approximate the $S U(3)$ Wilson action by a modified action based on a subgroup [33,35,36,46,49-52], although only in [33] were Monte Carlo calculations undertaken. Intuitively, the addition of further terms in the action of the discrete group that are proportional in the continuum to $\operatorname{Tr} F_{\mu \nu} F^{\mu \nu}$, but take different values, changes the values that $S$ can take. This lowers the gap $\delta S$ between the lowest actions and corresponds to a closer approach to the continuum.

In [33], simulations using $S(648)$ with a Wilson action modified by a $\left|\operatorname{Tr} U_{p}\right|^{2}$ term (equivalent to the trace in the adjoint representation) were performed. Even with this modified action $S(648)$ was inadequate to reach the scaling regime. Further, it was conjectured, based on small-scale simulations and mean-field estimates, that $S(1080)$ would also fail to reach the scaling regime with that modified action. Calculations with modified actions of $S U(3)$ were also performed to study thermodynamics and reduce lattice spacing errors [53-57].

\section{RESULTS}

With this history in mind, we study the viability of $S(1080)$ with a different action

$$
S=-\sum_{p}\left(\frac{\beta_{0}}{3} \operatorname{Re} \operatorname{Tr} U_{p}+\beta_{1} \operatorname{Re} \operatorname{Tr} U_{p}^{2}\right),
$$

where $U_{p} \in S(1080)$ indicates a plaquette, and the first term has been normalized such that, for $\beta_{1}=0$, the action matches the $S U(3)$ Wilson action (with $\beta=\beta_{0}$ ). Simulations can be performed efficiently by employing precomputed multiplication and trace tables.

One could argue heuristically that the action in Eq. (1) will lead to the same continuum limit as, for instance, the Wilson action of $S U(3)\left(S_{S U(3)}=-\frac{\beta_{S U(3)}}{3} \sum_{p} \operatorname{ReTr} U_{p}\right)$ by noting that the continuum limit in asymptotically free theories is obtained by setting the coupling to be small ( $\beta$ large) and that in this limit only small field fluctuations are important. For small values of the field $S$ and $S_{S U(3)}$ agree as long as $\beta_{S U(3)}$ is a certain linear combination of $\beta_{0}$, $\beta_{1}$. The flaw with this argument is that as $\beta_{S U(3)}$ is increased toward the continuum limit, so do $\beta_{0}, \beta_{1}$ and, at some point, the fluctuations become smaller than the separation between the identity and the nearest element to it in $S(1080)$ and fields freeze. Beyond this point it makes no sense to consider small fluctuations in the $S(1080)$ theory. This is seen dramatically in Fig. 1 where the average 


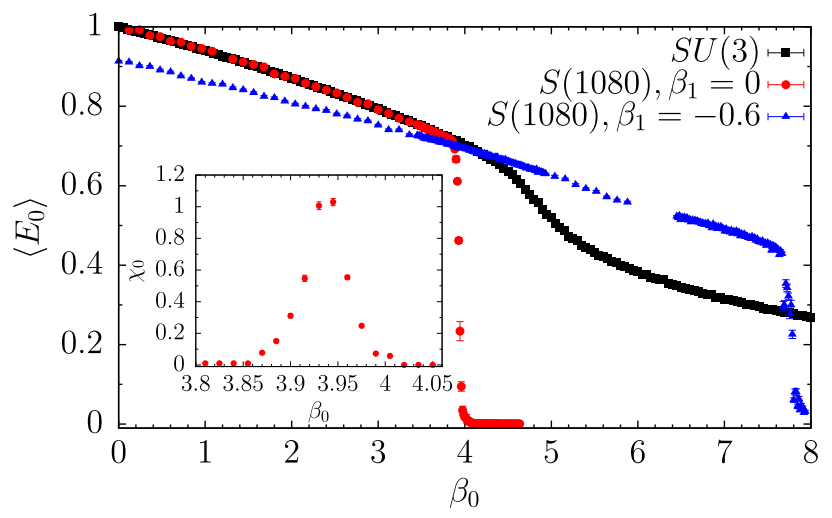

FIG. 1. Average energy per plaquette, $\left\langle E_{0}\right\rangle=1-\operatorname{Re}\left\langle\operatorname{Tr} U_{p}\right\rangle / 3$, vs $\beta_{0}$ on $2^{4}$ lattice for (filled black square) $S U(3)$ and $S(1080)$ with (filled red circle) $\beta_{1}=0$ and with (filled blue up-pointing triangle) $\beta_{1}=-0.6$. (Inset) $\chi_{0}$ vs $\beta_{0}$ for $\beta_{1}=0$.

plaquette of the Wilson action $S U(3)$ theory (black) and the $S(1080)$ action $S$ at $\beta_{1}=0$ (red) are shown to agree very well until they abruptly diverge when freezing occurs. This agreement is expected since the strong coupling expansion predicts the difference in the average plaquette for $S U(3)$ and $S(1080)$ with $\beta_{1}=0$ to be of order $\mathcal{O}\left(10^{-6} \beta^{5}\right)$ [31]. By setting $\beta_{1}<0$ the gap between the two lowest action values is reduced and freezing occurs only at large $\beta_{0}$ (see blue curve in Fig. 1). Our proposal is to find a trajectory in the $\left(\beta_{0}, \beta_{1}\right)$ plane that avoids freezing. The idea is that as we move on this trajectory toward larger values of $\beta_{0}$ we produce configurations with larger correlation lengths, ideally increasing all the way to infinity. While we do not anticipate reaching infinite correlation length, within the scaling regime the correlation length grows rapidly which naively would reduce the discrepancies between $S(1080)$ and $S U(3)$ if the action generates the same physics. We must check, though, that this action generates the same physics as $S U(3)$.

Because we have complete freedom in choosing our trajectory other than it must avoid the freezing transition, we need only to roughly map out the phase diagram of the $S(1080)$ theory in the $\left(\beta_{0}, \beta_{1}\right)$ plane to determine where the theory is frozen and not useful to approximate $S U(3)$. Since these phases are not the emphasis of this work, and we only need to know the approximate location to define a trajectory, we obtained the rough phase diagram for a small $2^{4}$ lattice. Further, the exact identification of this boundary is complicated because each phase exists as a metastable state throughout the phase diagram and therefore critical slowing down occurs. While the exact location of the freezing transition may differ due to finite-size effects on such a small lattice, we mitigate this concern by choosing a trajectory conservatively far away from the transition line, and monitoring that no freeze-out occurs in our larger lattice ensembles.

To deal with this metastability and the associated long autocorrelation times, we used a parallel tempering algorithm similar to $[58,59]$. We perform simulations with a set of $\left\{\beta_{0, i}\right\}$ for a fixed $\beta_{1}$. For every fifth local update, the configuration with $\beta_{0, i}$ is randomly selected and swapped with the ensemble with $\beta_{0, j}$ where $j=i \pm 1, i \pm 2$ with probability:

$$
P_{i j}\left(\phi_{i}, \phi_{j}\right)=\min \left(1, e^{\left(\beta_{0, i}-\beta_{0, j}\right)\left(\tilde{S}\left[\phi_{i}\right]-\tilde{S}\left[\phi_{j}\right]\right)}\right) .
$$

With this algorithm, we were able to map out the full $\left(\beta_{0}, \beta_{1}\right)$ space by searching for peaks in the susceptibilities $\chi_{0}=\frac{\partial^{2}\langle S\rangle}{\partial \beta_{0}^{2}}$ and/or $\chi_{1}=\frac{\partial^{2}\langle S\rangle}{\partial \beta_{1}^{2}}$ (see Fig. 2). Besides the freezing transition (shown in red), there are additional transitions shown in the upper left corner of the phase diagram delineating regions where the dynamics is partially frozen down to a subset of the group elements. This rich structure is qualitatively similar to the one found in the $S U(3)$ theory but it is of little concern to us. We focus instead on the lower $\left(\beta_{1}<0\right)$ region of the phase diagram. At $\beta_{1}=0$ we measure $\beta_{c}=3.935(5)$, a larger value than the $\beta_{c}=3.58(2)$ found with smaller statistics in [31].

With the freezing transition mapped out, we can propose a way to approach the continuum limit by performing simulations along a trajectory that avoids the frozen phase. We choose the trajectory

$$
\beta_{1}=-0.1267 \beta_{0}+0.253
$$

shown as a dotted line in Fig. 2 which is parallel to the freezing transition at large $\beta_{0}, \beta_{1}$.

From perturbative lattice $S U(3)$ arguments, a line of constant continuum coupling $1 / g^{2}$ should have a certain slope in the $\beta_{0}, \beta_{1}$ plane analogous to that derived in [31] for a different modified action. If these arguments are valid for our $S(1080)$ action, there is a similar slope in our coupling plane. Furthermore, the border of the freezing region is a line at large values of $\beta_{0}$. We chose our

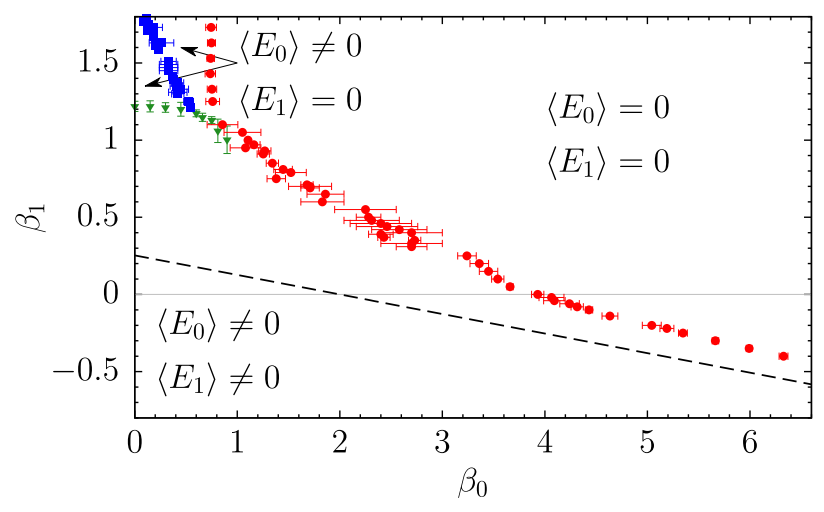

FIG. 2. Phase diagram in the $\left(\beta_{0}, \beta_{1}\right)$ plane for a $2^{4}$ lattice where $\left\langle E_{1}\right\rangle=1-\operatorname{Re}\left\langle\operatorname{Tr} U_{p}^{2}\right\rangle / 3$ is an additional order parameter. (filled blue square) and (filled red circle) correspond to peaks in $\chi_{0}$ and (filled green down-pointing triangle) to peaks in $\chi_{1}$. Also shown as a dashed line is the trajectory used for exploring larger lattices. 
parameter line to asymptote parallel to the freezing line. We find indeed that along this line the lattice spacing approaches a constant, suggesting that the freezing line is parallel to the constant $g$ line for our action. Adding other terms to the action e.g., including fermions or Symanzik improvement would require recomputing the freezing transition and thus a new trajectory would have to be determined.

A pure gauge theory (without matter) can predict only ratios of observables. So, one observable should be used to set the scale, that is, to determine the dimensionful quantity $a$ at a fixed $\beta_{0}, \beta_{1}$. After this, the theory can make predictions for all other observables. In order to quantify the approach to the continuum and compare it with the expected continuum result we then need a minimum of two observables: one to set the scale and another to be predicted. We choose the pseudocritical temperature $T_{c}$ for deconfinement and the scale $t_{0}$ defined by the Wilson flow [60].

We determine $T_{c}$ by looking at the distribution of values of the Polyakov loop $P$. In the confined phase they concentrate around zero, while at higher temperatures they cluster around the vertices of a triangle. We denote by $w_{c}$, $w_{3}$ the number of configurations in which $P$ lies near zero or near one of the vertices of the triangle. Following the procedure outlined in [61], we label as "center" configurations the ones that lie inside an equilateral triangle centered at the origin and rotated such that one side is perpendicular to the positive real axis. The separatrix is adjusted to the minimum of the histogram of the $\operatorname{Re} \operatorname{Tr} P$. This position is determined from simulations close to the transition point. There is some ambiguity in this definition but we include this variability into our error budget. We then look at the quantity $s\left(\beta_{0}, \beta_{1}\right)=\frac{3 w_{c}-w_{3}}{3 w_{c}+w_{3}}$ : It is +1 deep in the confined phase and reaches -1 deep in the deconfined phase. When $s=0$, the theory is tuned to $T_{c}$. This definition of $T_{c}$ has been shown to have finite-volume effects that scale exponentially in the spatial volume for $S U(3)$, unlike the peak of $\chi_{0}$ which exhibits power law volume corrections [61]. We found that for $N_{t}=4$, the difference in $\left(\beta_{0}^{c}, \beta_{1}^{c}\right)$ for $N_{s}=12,16$ was negligible, confirming the same behavior in $S(1080)$. Therefore, we assumed $N_{s}=3 N_{t}$ has negligible finite-volume effects at larger $N_{t}$ and use this volume to compute $T_{c}$. For each set of parameters we collected $\mathcal{O}\left(10^{6}\right)$ measurements separated by ten sweeps. To perform a sweep, we visit each link and update it using a multihit Metropolis step.

For $N_{t}=4,6,8$ we scan $\left(\beta_{0}, \beta_{1}\right)$ along the trajectory Eq. (3) to find $\left(\beta_{0}^{c}, \beta_{1}^{c}\right)$ for which $s=0$. The values obtained are listed in Table I. For each of these the inverse physical temperature $1 / T=N_{t} a\left(\beta_{0}^{c}, \beta_{1}^{c}\right)=1 / T_{c}$ is the same.

We compute the scale $t_{0}$ defined by the Wilson flow for these sets of $\left(\beta_{0}^{c}, \beta_{1}^{c}\right)$ parameters. We first generate configurations on lattices of size $\left(3 N_{t}\right)^{4}$ where $N_{t}$ is the temporal size used to determine $T_{c}$. For each ensemble we
TABLE I. Wilson-flow parameters $\sqrt{t_{0}} / a$ and $\sqrt{t_{0.2}} / a$ found on lattices of size $\left(3 N_{t}\right)^{4}$ along our trajectory where $N_{t}$ is temporal size used to determine $T_{c}$. In the last two columns, the first error is statistical, and the second is from the separatrix.

\begin{tabular}{lcclcl}
\hline \hline$N_{t}$ & $N_{s}$ & $\beta_{0}^{c}$ & \multicolumn{1}{c}{$\beta_{1}^{c}$} & \multicolumn{1}{c}{$\sqrt{t_{0}} / a$} & \multicolumn{1}{c}{$\sqrt{t_{0.2}} / a$} \\
\hline 4 & 12 & $9.154(2)$ & $-0.9061(3)$ & $1.016(3)(3)$ & $0.8316(12)(20)$ \\
6 & 18 & $12.795(9)$ & $-1.3673(11)$ & $1.508(3)(5)$ & $1.2068(18)(42)$ \\
8 & 24 & $19.61(4)$ & $-2.231(5)$ & $2.000(4)(8)$ & $1.595(3)(6)$ \\
\hline \hline
\end{tabular}

generate 200 configurations, separated by 1000 sweeps. The configurations generated are represented as $S U(3)$ matrices and used as initial conditions for performing the Wilson flow [60]:

$$
\begin{aligned}
\dot{V}_{t}(x, \mu) & =-\frac{1}{\beta_{0}}\left(\partial_{x, \mu} S_{W}\left[V_{t}\right]\right) V_{t}(x, \mu), \\
\left.V_{t}(x, \mu)\right|_{t=0} & =U(x, \mu)
\end{aligned}
$$

where $S_{W}\left[V_{t}\right]$ is the Wilson action of $S U(3)$ fields $V_{t}$ at some Wilson-flow time $t$. Using the flow, we define two observables $t_{X=0.2}$ and $t_{X=0.3}$ implicitly by the expression

$$
\left(t^{2}\langle E\rangle\right)_{t=t_{X}}=X
$$

where $X=0.2,0.3$, and $E$ is the lattice clover definition of the energy density. Following the convention, $t_{X=0.3}$ is called $t_{0}$. Both of these observables have been measured precisely for $S U(3)$ pure gauge theory, allowing for comparison. By also measuring $t_{0.2}$, we probe higher energy scales where larger discrepancies between $S(1080)$ and $S U(3)$ should be found. Our results are found in Table I.

In the absence of discretization effects, the value of $t_{0}$ in physical units should be the same on all our lattices. We demonstrate that the variation of $t_{0}$ as we approach the continuum is mild and the extrapolated value agrees with the full $S U(3)$ result. With our data, it is possible to construct a dimensionless quantity, $T_{c} \sqrt{t_{X}}$ which can be compared to those of $S U(3)$ at both finite lattice spacing $a$ and by extrapolating to the continuum. Using a linear extrapolation, we compute a continuum value of $T_{c} \sqrt{t_{0}}=$ $0.2489(11)$ which is in agreement with $T_{c} \sqrt{t_{0}}=$ 0.2489 (14) [61] and $T_{c} \sqrt{t_{0}}=0.2473(7)$ [62] computed in full $S U(3)$. Similarly, our extrapolated value of $\frac{\sqrt{t_{0}}}{\sqrt{t_{0.2}}}=$ $0.1269(6)$ is in good agreement with the value of 0.1264(4) computed for $S U(3)$ [63]. Our results for $T_{c} \sqrt{t_{0}}$ are compared to [61] in Fig. 3. It is interesting to note that the $\mathcal{O}\left(a^{2}\right)$ corrections appear milder for the modified action used here compared to the Wilson action of the $S U(3)$. This feature of modified actions has been observed previously in $S U(3)$ [53-57], which suggest further benefits of using this action for quantum simulations. 

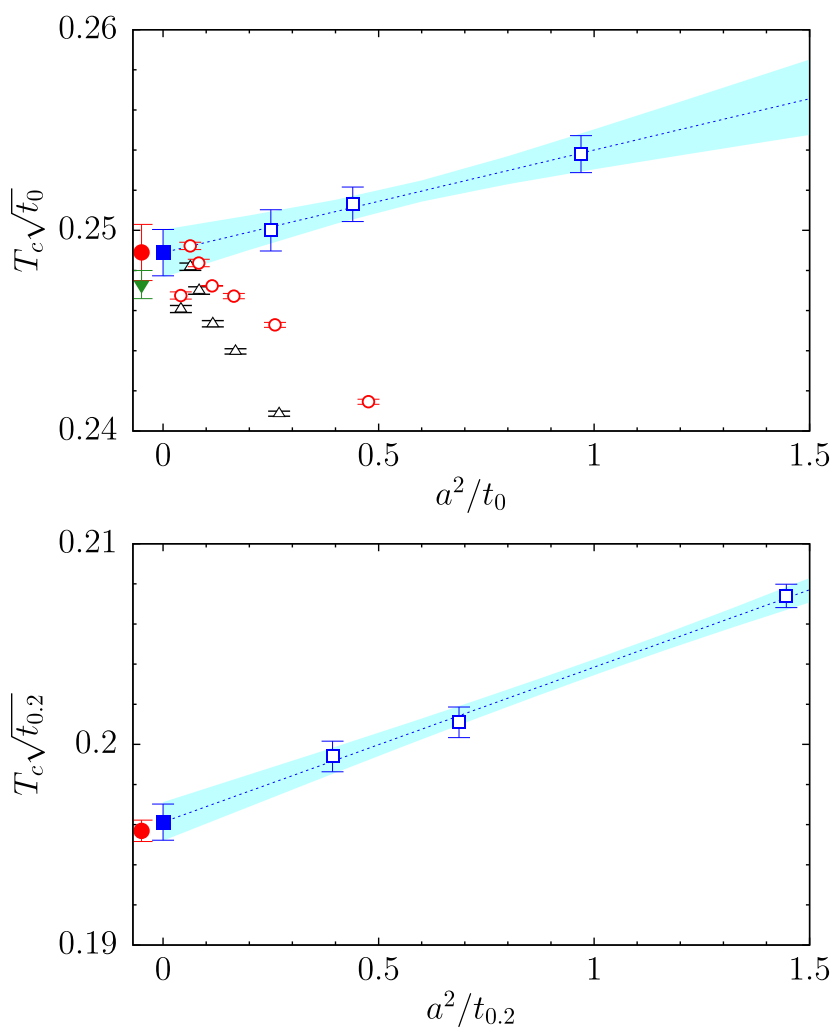

FIG. 3. (Top) $T_{c} \sqrt{t_{0}}$ vs $a^{2} / t_{0}$. Our results (blue square) compared to $S U(3)$ results from [61] using Wilson (black uppointing triangle) and Wilson-improved (red circle) energies are reproduced for comparison. Our extrapolated value (filled blue square) is compared to $S U(3)$ results of [61] (filled red circle) and [62] (filled green down-pointing triangle). (Bottom) $T_{c} \sqrt{t_{0.2}}$ vs $a^{2} / t_{0.2}$ and the extrapolation compared with the results of [63] with same symbols.

Although the $S(1080)$ action may be inequivalent to $S U(3)$ in the continuum limit, it is similar at finite $a$ and by making a connection to $S U(3)$, we will derive physical values for $t_{0}$ and a lattice spacing $a$ for each of our ensembles. Using the $S U(3)$ relations of $[62,63]$ between $w_{0.4}, T_{c}, \Lambda_{S U(3)}$, and $r_{0}=0.49(4) \mathrm{fm}$, we obtain $\sqrt{t_{0}} \approx$ $0.16(2) \mathrm{fm}$ where our error is dominated by $r_{0}$, agreeing with $\sqrt{t_{0}}=0.1638(10) \mathrm{fm}$ determined in $S U(3)$ [64]. With this, our $24^{4}$ lattice has $a \approx 0.08 \mathrm{fm}$ or $2.5 \mathrm{GeV}^{-1}$. This suggests it would be possible to extract and compare glueball states $[65,66]$ or quenched calculations of hadron masses [67] to $S U(3)$ values with subpercent precision.

\section{DISCUSSION AND CONCLUSIONS}

In this work, we have presented results for the discrete gauge group $S(1080)$ using a modified Wilson action. After mapping the entire phase diagram of the new action, we have found a parameter trajectory that avoids the freezing transition, allowing for calculations with lattice spacing $a \approx 0.08 \mathrm{fm}$. We have shown that this action is capable of reproducing the physics of $S U(3)$ below $2.5 \mathrm{GeV}^{-1}$ by measuring $T_{c} \sqrt{t_{0}}=0.2489(11)$ and $\frac{\sqrt{t_{0}}}{\sqrt{t_{0.2}}}=0.1269(6)$, which agree to remarkable precision with the full group.

The qubit savings from using $S(1080)$ instead of $S U(3)$ are dramatic. In the NISQ era, where small lattice sizes and noisy gates will likely dominate the error, the parameters used in this paper should be a sufficient approximation of $S U(3)$. However, if gluon actions are required at $a<0.08 \mathrm{fm}$, the action in Eq. (1) on the trajectory specified by Eq. (3) may be insufficient since $a$ on this trajectory seems to have a minimum. Another trajectory nearer to the freezing transition may provide a smaller lattice spacing. Future work is required to determine whether we can generate arbitrarily small $a$ on a different trajectory with our action or if additional terms are required. Guidance for which terms could be included could be taken from the cumulant expansion of $S U(3)$ in the $S(1080)$ terms previously studied $[35,36]$. Furthermore, questions of how well other observables like hadronic spectra are reproduced is left for future studies. Previous work with $S U(2)$ has demonstrated that coupling fermions to discrete subgroups can be done in the same way as continuous groups $[47,48]$. Given that circuit depth is of concern in the NISQ era, a broad search in modified action space should be undertaken with an eye toward terms that require few quantum gates while still efficiently reaching the scaling regime of $S U(3)$. Quantitative comparisons of these actions will require constructing the primitive quantum gates for $S(1080)$ along the lines of [44] where smaller discrete groups were investigated. This is an ongoing work. At that point, it will be possible to compare the feasibility of this method with different actions as well as to quantum link model methods being studied on quantum simulators [68-71]. It should be noted that inclusion of the $\operatorname{Re} \operatorname{Tr} U_{p}^{2}$ will require additional circuit depth, and no new primitive quantum gates are required.

\section{ACKNOWLEDGMENTS}

The authors thank Y. Yamauchi and Y. Cai for insight into quantum gates. A. A. is supported in part by the National Science Foundation CAREER Grant No. PHY1151648 and by the U.S. Department of Energy under Contract No. DE-FG02-95ER-40907. A. A. acknowledges the hospitality of the University of Maryland where part of this work was performed. P. B., S. H., H. L., and S. L. are supported by the U.S. Department of Energy under Contract No. DE-FG02-93ER-40762. H. L. acknowledges the hospitality of U.W. where parts of this work were produced. 
[1] R. P. Feynman, Int. J. Theor. Phys. 21, 467 (1982).

[2] P. Jordan and E. P. Wigner, Z. Phys. 47, 631 (1928).

[3] F. Verstraete and J. I. Cirac, J. Stat. Mech. (2005) P09012.

[4] E. Zohar and J. I. Cirac, Phys. Rev. B 98, 075119 (2018).

[5] J. D. Whitfield, V. Havlíček, and M. Troyer, Phys. Rev. A 94, 030301 (2016).

[6] E. A. Martinez et al., Nature (London) 534, 516 (2016).

[7] N. Klco, E. F. Dumitrescu, A. J. McCaskey, T. D. Morris, R. C. Pooser, M. Sanz, E. Solano, P. Lougovski, and M. J. Savage, Phys. Rev. A 98, 032331 (2018).

[8] H. Lamm and S. Lawrence, Phys. Rev. Lett. 121, 170501 (2018).

[9] O. Shehab, K. A. Landsman, Y. Nam, D. Zhu, N. M. Linke, M. J. Keesan, R. C. Pooser, and C. R. Monroe, arXiv:1904 .04338 .

[10] K. Kasamatsu, I. Ichinose, and T. Matsui, Phys. Rev. Lett. 111, 115303 (2013).

[11] E. Zohar, J. I. Cirac, and B. Reznik, Rep. Prog. Phys. 79, 014401 (2016).

[12] D. C. Hackett, K. Howe, C. Hughes, W. Jay, E. T. Neil, and J. N. Simone, Phys. Rev. A 99, 062341 (2019).

[13] A. Macridin, P. Spentzouris, J. Amundson, and R. Harnik, Phys. Rev. Lett. 121, 110504 (2018).

[14] K. Yeter-Aydeniz, E. F. Dumitrescu, A. J. McCaskey, R. S. Bennink, R. C. Pooser, and G. Siopsis, Phys. Rev. A 99, 032306 (2019).

[15] N. Klco and M. J. Savage, Phys. Rev. A 99, 052335 (2019).

[16] A. Bazavov, Y. Meurice, S.-W. Tsai, J. Unmuth-Yockey, and J. Zhang, Phys. Rev. D 92, 076003 (2015).

[17] J. Zhang, J. Unmuth-Yockey, J. Zeiher, A. Bazavov, S. W. Tsai, and Y. Meurice, Phys. Rev. Lett. 121, 223201 (2018).

[18] J. F. Unmuth-Yockey, Phys. Rev. D 99, 074502 (2019).

[19] J. Unmuth-Yockey, J. Zhang, A. Bazavov, Y. Meurice, and S.-W. Tsai, Phys. Rev. D 98, 094511 (2018).

[20] T. V. Zache, F. Hebenstreit, F. Jendrzejewski, M. K. Oberthaler, J. Berges, and P. Hauke, Sci. Technol. 3, 034010 (2018).

[21] I. Raychowdhury and J. R. Stryker, arXiv:1812.07554.

[22] D. B. Kaplan and J. R. Stryker, arXiv:1806.08797.

[23] J. R. Stryker, Phys. Rev. A 99, 042301 (2019).

[24] A. Alexandru, P. F. Bedaque, H. Lamm, and S. Lawrence (NuQS Collaboration), Phys. Rev. Lett. 123, 090501 (2019).

[25] S. Chandrasekharan and U. J. Wiese, Nucl. Phys. B492, 455 (1997).

[26] B. Schlittgen and U. J. Wiese, Phys. Rev. D 63, 085007 (2001).

[27] R. Brower, S. Chandrasekharan, and U. J. Wiese, Phys. Rev. D 60, 094502 (1999).

[28] B. B. Beard, M. Pepe, S. Riederer, and U. J. Wiese, Phys. Rev. Lett. 94, 010603 (2005).

[29] D. Petcher and D. H. Weingarten, Phys. Rev. D 22, 2465 (1980).

[30] L. Jacobs and C. Rebbi, J. Comput. Phys. 41, 203 (1981).

[31] G. Bhanot and C. Rebbi, Phys. Rev. D 24, 3319 (1981).

[32] H. Grosse and H. Kuhnelt, Phys. Lett. 101B, 77 (1981).

[33] G. Bhanot, Phys. Lett. 108B, 337 (1982).
[34] P. Lisboa and C. Michael, Phys. Lett. 113B, 303 (1982).

[35] H. Flyvbjerg, Nucl. Phys. B243, 350 (1984).

[36] H. Flyvbjerg, Nucl. Phys. B240, 481 (1984).

[37] E. Zohar, J. I. Cirac, and B. Reznik, Phys. Rev. A 88, 023617 (2013).

[38] P. Hasenfratz and F. Niedermayer, Proc. Sci., HEP2001 (2001) 229 [arXiv:hep-lat/0112003].

[39] S. Caracciolo, A. Montanari, and A. Pelissetto, Phys. Lett. B 513, 223 (2001).

[40] P. Hasenfratz and F. Niedermayer, Nucl. Phys. B, Proc. Suppl. 94, 575 (2001).

[41] A. Patrascioiu and E. Seiler, Phys. Rev. E 57, 111 (1998).

[42] R. Krcmar, A. Gendiar, and T. Nishino, Phys. Rev. E 94, 022134 (2016).

[43] S. Caracciolo, A. Montanari, and A. Pelissetto, Phys. Lett. B 513, 223 (2001).

[44] H. Lamm, S. Lawrence, and Y. Yamauchi (NuQS Collaboration), Phys. Rev. D 100, 034518 (2019).

[45] M. Creutz, L. Jacobs, and C. Rebbi, Phys. Rev. D 20, 1915 (1979).

[46] M. Creutz and M. Okawa, Nucl. Phys. B220, 149 (1983).

[47] D. H. Weingarten and D. N. Petcher, Phys. Lett. 99B, 333 (1981).

[48] D. Weingarten, Phys. Lett. 109B, 57 (1982).

[49] R. C. Edgar, Nucl. Phys. B200, 345 (1982).

[50] M. Fukugita, T. Kaneko, and M. Kobayashi, Nucl. Phys. B215, 289 (1983).

[51] D. Horn, M. Karliner, E. Katznelson, and S. Yankielowicz, Phys. Lett. 113B, 258 (1982).

[52] C. Ayala and M. Baig, Ann. Phys. (N.Y.) 198, 1 (1990).

[53] T. Blum, C. E. DeTar, U. M. Heller, L. Karkkainen, K. Rummukainen, and D. Toussaint, Nucl. Phys. B442, 301 (1995).

[54] U. M. Heller, Phys. Lett. B 362, 123 (1995).

[55] U. M. Heller, Nucl. Phys. B, Proc. Suppl. 47, 262 (1996).

[56] M. Hasenbusch and S. Necco, Nucl. Phys. B, Proc. Suppl. 140, 743 (2005).

[57] M. Hasenbusch and S. Necco, J. High Energy Phys. 08 (2004) 005.

[58] R. H. Swendsen and J.-S. Wang, Phys. Rev. Lett. 57, 2607 (1986).

[59] D. J. Earl and M. W. Deem, Phys. Chem. Chem. Phys. 7, 3910 (2005).

[60] M. Luscher, J. High Energy Phys. 08 (2010) 071; 03 (2014) 092(E).

[61] A. Francis, O. Kaczmarek, M. Laine, T. Neuhaus, and H. Ohno, Phys. Rev. D 91, 096002 (2015).

[62] M. Kitazawa, T. Iritani, M. Asakawa, T. Hatsuda, and H. Suzuki, Phys. Rev. D 94, 114512 (2016).

[63] M. Asakawa, T. Hatsuda, T. Iritani, E. Itou, M. Kitazawa, and H. Suzuki, arXiv:1503.06516.

[64] R. Sommer, Proc. Sci., LATTICE2013 (2014) 015 [arXiv: 1401.3270].

[65] C. J. Morningstar and M. J. Peardon, Phys. Rev. D 60, 034509 (1999).

[66] Y. Chen et al., Phys. Rev. D 73, 014516 (2006).

[67] S. Aoki et al. (CP-PACS Collaboration), Phys. Rev. D 67, 034503 (2003). 
[68] D. Banerjee, M. Dalmonte, M. Muller, E. Rico, P. Stebler, U. J. Wiese, and P. Zoller, Phys. Rev. Lett. 109, 175302 (2012).

[69] D. Banerjee, M. Bögli, M. Dalmonte, E. Rico, P. Stebler, U. J. Wiese, and P. Zoller, Phys. Rev. Lett. 110, 125303 (2013).
[70] D. Marcos, P. Widmer, E. Rico, M. Hafezi, P. Rabl, U. J. Wiese, and P. Zoller, Ann. Phys. (Amsterdam) 351, 634 (2014).

[71] E. Rico, M. Dalmonte, P. Zoller, D. Banerjee, M. Bögli, P. Stebler, and U. J. Wiese, Ann. Phys. (Amsterdam) 393, 466 (2018). 